\title{
Fetal neuroimaging: US and MRI
}

\section{Elida Vazquez • Nuria Mayolas • Ignacio Delgado • \\ Teresa Higueras}

Published online: 7 July 2009

(C) Springer-Verlag 2009

\section{Erratum to: Pediatr Radiol}

\section{DOI 10.1007/s00247-009-1221-x}

The legends of Figs. 7-11 were wrongly assigned. These figures are reproduced here, each with the correct legend.

The online version of the original article can be found at http://dx.doi. org/10.1007/s00247-009-1221-x

E. Vazquez $(\bowtie) \cdot N$. Mayolas $\cdot$ I. Delgado

Department of Paediatric Radiology,

Hospital Universitario Vall d'Hebron,

Psg. Vall d'Hebron, 112-119,

08035 Barcelona, Spain

e-mail: evazquez@vhebron.net

\section{T. Higueras}

Fetal Medicine Unit, Hospital Universitario Vall d'Hebron,

Barcelona, Spain 
Fig. 7 Mega cisterna magna. Sagittal (a) and axial (b) HASTE T2-W images in a 34 weeks' gestation age fetus demonstrate a large cisterna magna. The vermis is identified and is 7 normal in size
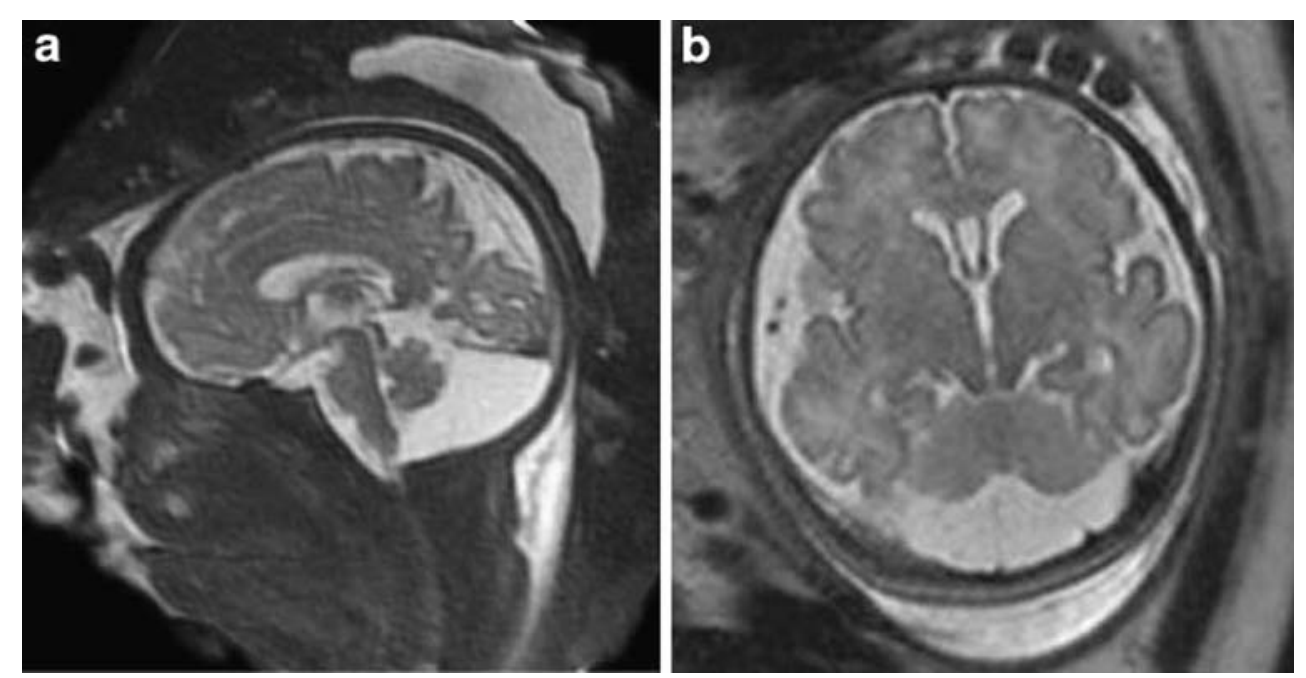
Fig. 8 Dandy-Walker malformation. Axial US (a) obtained at 30 weeks' gestation, and axial (b) and sagittal (c) MR HASTE T2-weighted images in the same 32-gestational-week-old fetus, show an increased cystic retrocerebellar space with a severely hypoplastic cerebellum and hydrocephalus. Postnatal followup sagittal T1-W (d) and axial T2-W (e) images demonstrate an abnormal cerebral white matter not seen in the prenatal MR examination
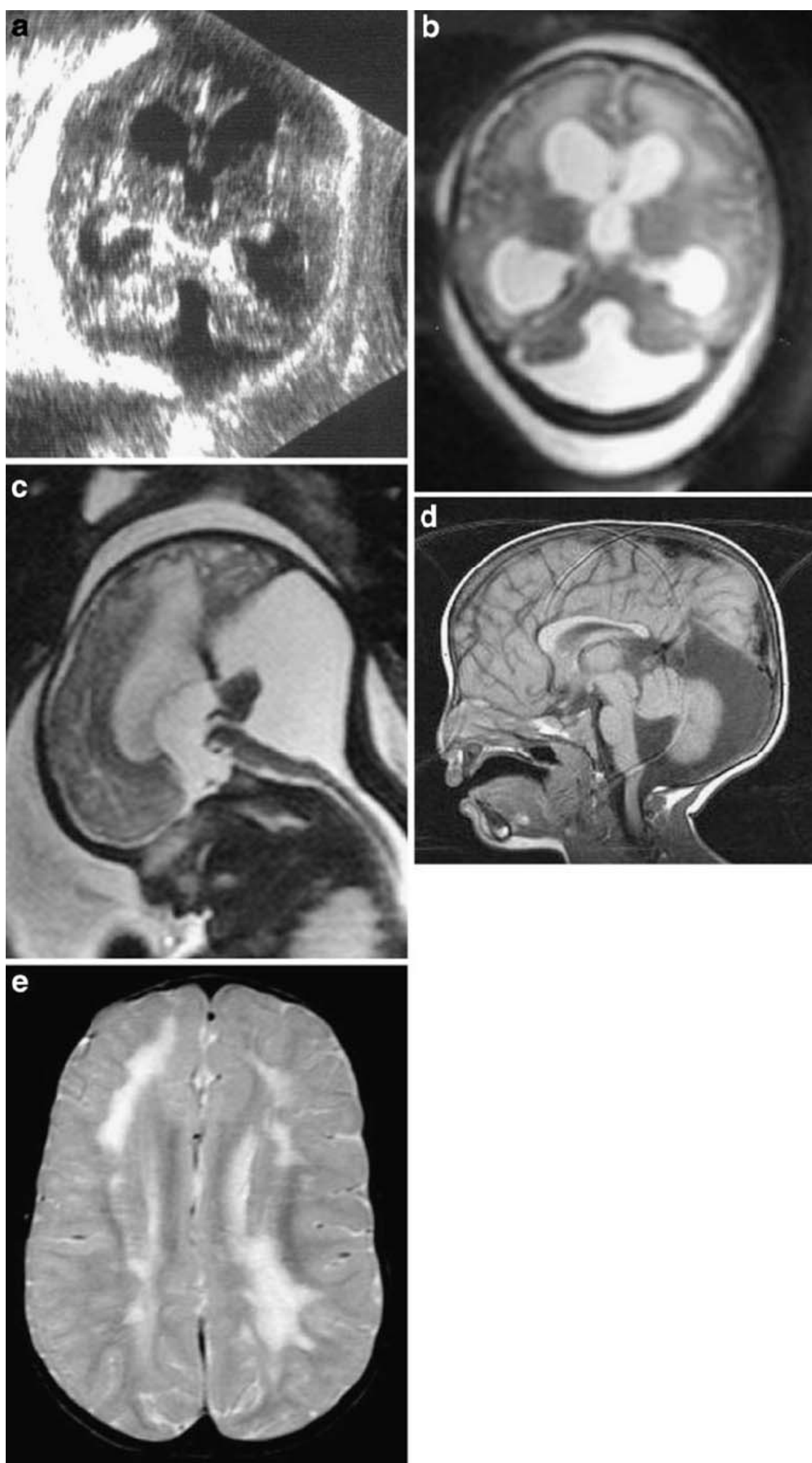

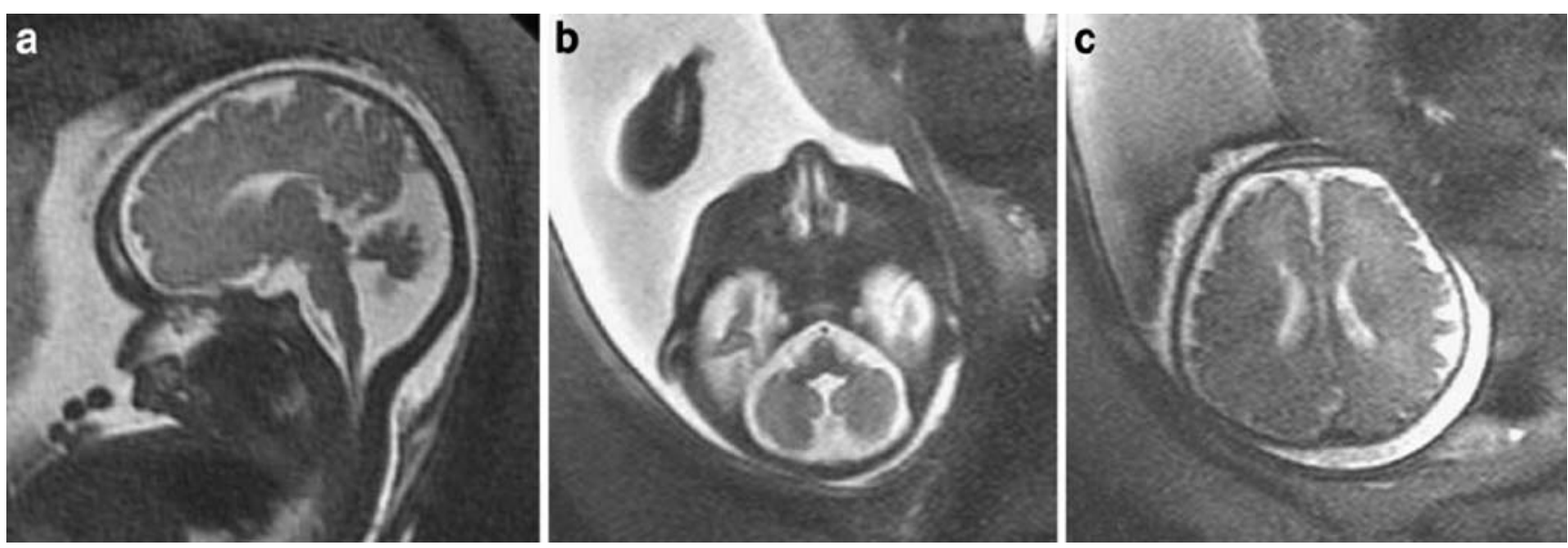

Fig. 9 Dandy-Walker variant. Sagittal (a) and axial (b) HASTE T2-W images in 33-gestational-week-old fetus demonstrate vermian hypoplasia and normal posterior fossa size. Supratentorial structures are normal (c)

Fig. 10 Cerebellar vermian hypoplasia. Sagittal (a) and axial (b) HASTE T2-W images in a 20 weeks' gestation age fetus show a small inferior vermis. Note also the hypoplastic corpus callosum. The gestation was interrupted and a WolfHirschhorn (4p-) syndrome was eventually demonstrated
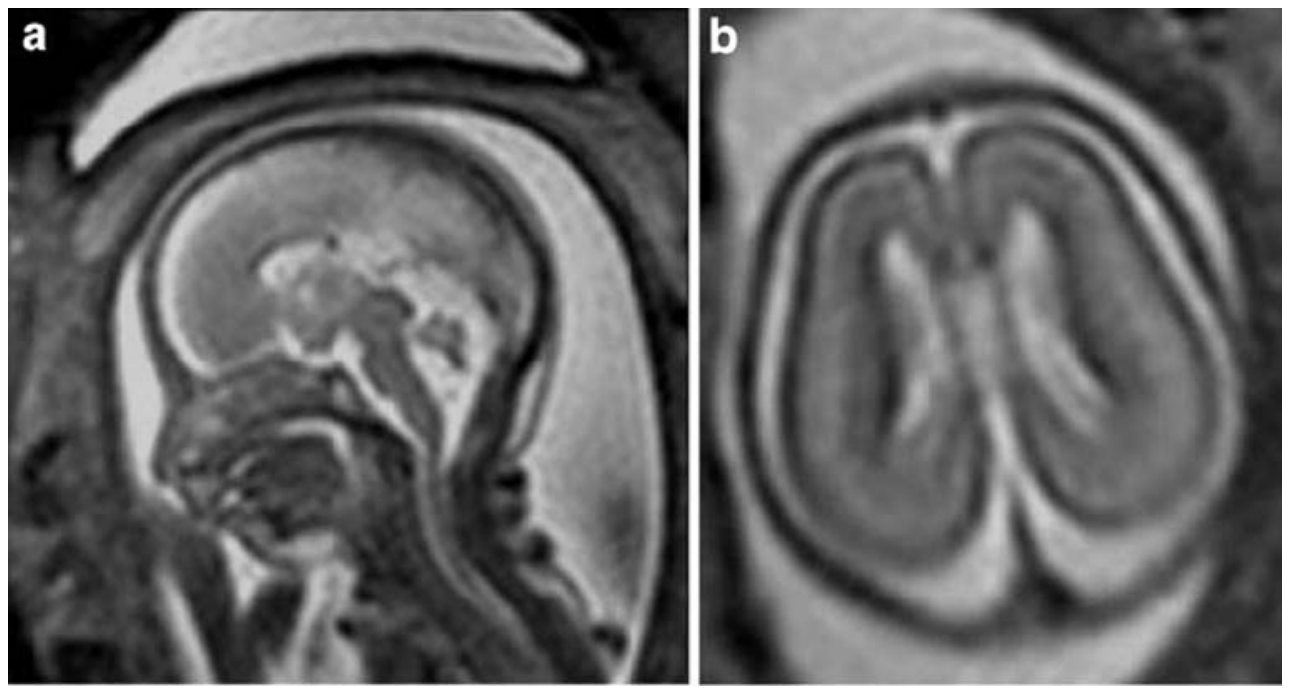
Fig. 11 Partial rhombencephalosynapsis. Axial (a) and sagittal (b) HASTE T2-W images in a 22 weeks' gestation age fetus show mild ventriculomegaly and abnormal cerebellar vermis. Follow-up MR imaging (c-e) performed at 31 weeks more clearly depicts abnormal inferior vermis and persistent mild ventriculomegaly
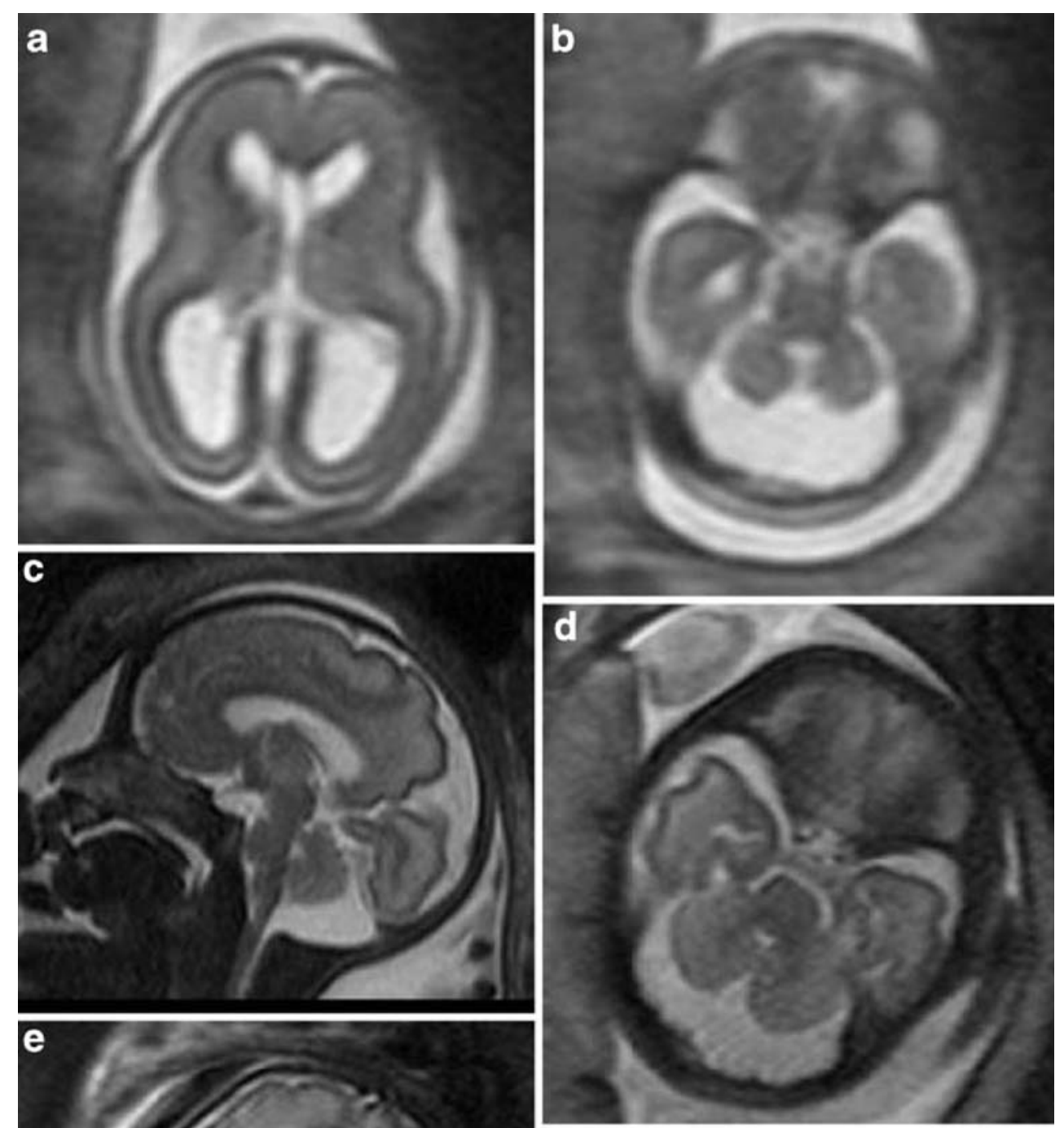

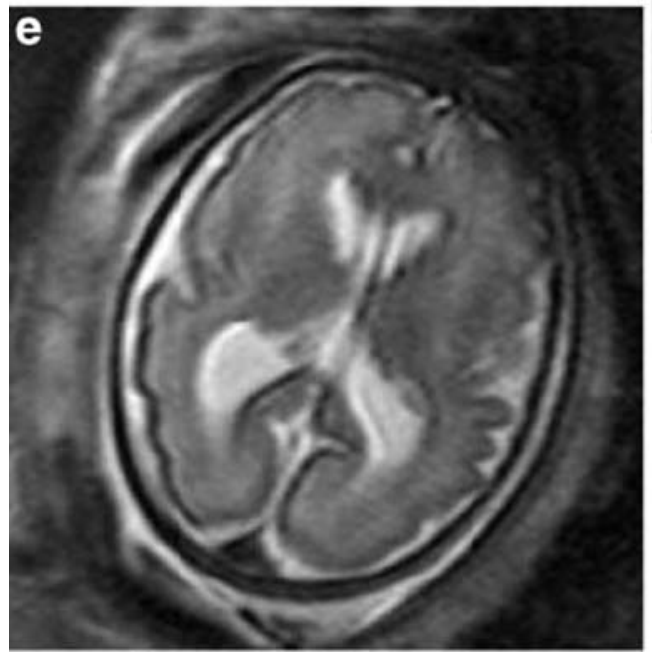

